\title{
A PAIR OF UNSYMMETRICAL FOURIER KERNELS
}

\author{
BY \\ ROOP N. KESARWANI $\left({ }^{1}\right)$
}

1. Introduction. The functions $k(x)$ and $h(x)$ are said [1, p.212] to form a pair of Fourier kernels if the reciprocal equations

$$
\begin{aligned}
& g(x)=\int_{0}^{\infty} k(x y) f(y) d y, \\
& f(x)=\int_{0}^{\infty} h(x y) g(y) d y
\end{aligned}
$$

are simultaneously satisfied. The kernels are said to be symmetrical if $k(x)$ $=h(x)$ and unsymmetrical if $k(x) \neq h(x)$. In an earlier paper [2, p.953] I found a pair of unsymmetrical Fourier kernels in terms of Meijer's $G$-functions. They are

$$
\begin{aligned}
& k(x)=2 \gamma x^{\gamma-1 / 2} G_{p+q, m+n}^{m, p}\left(\begin{array}{l|l}
x^{2 \gamma} & \begin{array}{l}
a_{1}, \cdots, a_{p}, b_{1}, \cdots, b_{q} \\
c_{1}, \cdots, c_{m}, d_{1}, \cdots, d_{n}
\end{array}
\end{array}\right), \\
& h(x)=2 \gamma x^{\gamma-1 / 2} G_{p+q, m+n}^{n, q}\left(\begin{array}{ll}
x^{2 \gamma} & \begin{array}{l}
-b_{1}, \cdots,-b_{q},-a_{1}, \cdots,-a_{p} \\
-d_{1}, \cdots,-d_{n},-c_{1}, \cdots,-c_{m}
\end{array}
\end{array}\right),
\end{aligned}
$$

where

$$
\gamma>0, n-p=m-q>0 \text { and }\left({ }^{2}\right) \quad \sum^{p} a_{j}+\sum^{q} b_{j}=\sum^{m} c_{j}+\sum^{n} d_{j} .
$$

I also established in later papers the reciprocity of (1.1) and (1.1') by using the ordinary convergence methods [ 3, p. 21] and the methods of mean square convergence [ 4, p. 275]. The object of the present paper is to obtain a pair of unsymmetric Fourier kernels of a form more general than the $G$-functions. Our kernels will be functions of the Mellin-Barnes type

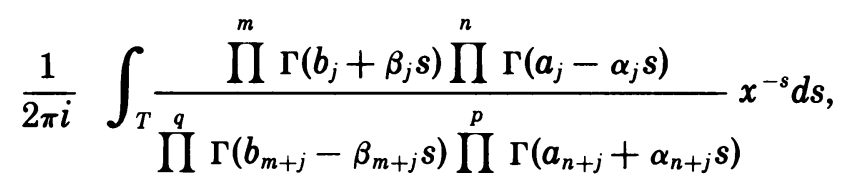

Received by the editors October 10, 1962 and, in revised form, November 18, 1963.

$\left({ }^{1}\right)$ Formerly Roop Narain.

(2) For the sake of convenience the sums $\sum_{j=1}^{p} f(j)$ and the products $I_{j=1}^{p}\left(G_{j}\right)$ in which the summation or the product starts from $j=1$ will be denoted by $\sum^{p} f(j)$ and $\operatorname{II}^{p}\left(G_{j}\right)$, respectively. 
where the parameters are such that the poles of the integrand are simple and those of $\Gamma\left(b_{j}+\beta_{j} s\right), j=1, \cdots, m$ lie on one side of the contour $T$ and those of $\Gamma\left(a_{j}-\alpha_{j} s\right), j=1, \cdots, n$ lie on the other side. This function was introduced by Fox [5, p. 408]. Except for a special case of this function studied by Boersma $[6$, p. 38], this function has not been studied to any great extent. This function has an advantage that for a suitable contour $T$, the Mellin transform of the function in (3.1) is evidently the coefficient of $x^{-s}$ in the integrand of (1.3). We, in fact, are required in our work to consider functions whose Mellin transforms satisfy a certain functional relation. We therefore consider the following functions

$$
\begin{aligned}
& H^{(1)}(x) \equiv \frac{1}{2 \pi i} \int_{T}^{\frac{\prod^{m} \Gamma\left(c_{j}+\gamma_{j}\left(s-\frac{1}{2}\right)\right) \prod^{p} \Gamma\left(a_{j}-\alpha_{j}\left(s-\frac{1}{2}\right)\right)}{\prod^{n} \Gamma\left(d_{j}-\delta_{j}\left(s-\frac{1}{2}\right)\right) \prod^{q} \Gamma\left(b_{j}+\beta_{j}\left(s-\frac{1}{2}\right)\right)}} x^{-s} d s, \\
& \left(1.4^{\prime}\right) H^{(2)}(x) \equiv \frac{1}{2 \pi i} \int_{T} \frac{\prod_{T}^{n} \Gamma\left(d_{j}+\delta_{j}\left(s-\frac{1}{2}\right)\right) \stackrel{q}{\prod} \Gamma\left(b_{j}-\beta_{j}\left(s-\frac{1}{2}\right)\right)}{\prod^{m} \Gamma\left(c_{j}-\gamma_{j}\left(s-\frac{1}{2}\right)\right) \stackrel{p}{\prod} \Gamma\left(a_{j}+\alpha_{j}\left(s-\frac{1}{2}\right)\right)} x^{-s} d s,
\end{aligned}
$$

where we make the following simplifying assumptions:

(i)

$$
\begin{gathered}
m-q=n-p>0 . \\
\alpha_{j}>0, \quad j=1, \cdots, p ; \quad \beta_{j}>0, \quad j=1, \cdots, q ; \\
\gamma_{j}>0, \quad j=1, \cdots, m ; \quad \delta_{j}>0, \quad j=1, \cdots, n . \\
\frac{1}{2} D=\sum^{m} \gamma_{j}-\sum^{q} \beta_{j}=\sum^{n} \delta_{j}-\sum^{p} \alpha_{j}>0 . \\
\sum^{m} c_{j}-\sum^{q} b_{j}=\sum^{n} d_{j}-\sum^{p} a_{j} .
\end{gathered}
$$

(v) All the poles of the integrand in (1.4) and (1.4') are simple.

(vi) The contour $T$ is a straight line parallel to the imaginary axis in the $s$-plane and the poles of $\Gamma\left(c_{j}+\gamma_{j}\left(s-\frac{1}{2}\right)\right)$ and $\Gamma\left(d_{j}+\delta_{j}\left(s-\frac{1}{2}\right)\right)$ lie on the left of $T$ while those of $\Gamma\left(a_{j}-\alpha_{j}\left(s-\frac{1}{2}\right)\right)$ and $\Gamma\left(b_{j}-\beta_{j}\left(s-\frac{1}{2}\right)\right)$ lie on the right of $T$.

We shall prove that $H^{(1)}(x)$ and $H^{(2)}(x)$ play the role of unsymmetrical Fourier kernels. Let us write (1.4) and (1.4') as

$$
H^{(1)}(x)=\frac{1}{2 \pi i} \int_{T} \mathfrak{S}^{(1)}(s) x^{-s} d s
$$




$$
H^{(2)}(x)=\frac{1}{2 \pi i} \int_{T} \mathfrak{S}^{(2)}(s) x^{-s} d s,
$$

where

$$
\begin{aligned}
\mathfrak{S}^{(1)}(s)= & \frac{\prod^{m} \Gamma\left(c_{j}+\gamma_{j}\left(s-\frac{1}{2}\right)\right) \prod^{p} \Gamma\left(a_{j}-\alpha_{j}\left(s-\frac{1}{2}\right)\right)}{\prod^{n} \Gamma\left(d_{j}-\delta_{j}\left(s-\frac{1}{2}\right)\right) \prod_{\prod}^{q} \Gamma\left(b_{j}+\beta_{j}\left(s-\frac{1}{2}\right)\right)}, \\
\mathfrak{S}^{(2)}(s)= & \frac{\prod^{n} \Gamma\left(d_{j}+\delta_{j}\left(s-\frac{1}{2}\right)\right) \prod^{q} \Gamma\left(b_{j}-\beta_{j}\left(s-\frac{1}{2}\right)\right)}{\prod^{m} \Gamma\left(c_{j}-\gamma_{j}\left(s-\frac{1}{2}\right)\right) \prod^{p} \Gamma\left(a_{j}+\alpha_{j}\left(s-\frac{1}{2}\right)\right)}
\end{aligned}
$$

Then $\mathfrak{S}^{(1)}(s)$ and $\mathfrak{S}^{(2)}(s)$ are Mellin transforms of $H^{(1)}(x)$ and $H^{(2)}(x)$, respectively.

In order that the functions $k(x)$ and $h(x)$ may be unsymmetrical Fourier kernels it is necessary $[1$, p. 214] that their Mellin transforms, $\kappa(s)$ and $\eta(s)$, respectively, should satisfy the functional relation

$$
\kappa(s) \eta(1-s)=1 .
$$

Clearly, $\mathfrak{S}^{(1)}(s)$ and $\mathfrak{W}^{(2)}(s)$ satisfy this functional relation and it is for this reason that the functions $H^{(1)}(x)$ and $H^{(2)}(x)$ have been chosen in this fashion. The satisfaction of $(1.8)$ alone by $\mathfrak{S}^{(1)}(s)$ and $\mathfrak{W}^{(2)}(s)$ is not sufficient to ensure the reciprocity

$$
\begin{aligned}
& g(x)=\int_{0}^{\infty} H^{(1)}(x y) f(y) d y, \\
& f(x)=\int_{0}^{\infty} H^{(2)}(x y) g(y) d y .
\end{aligned}
$$

More is required to be done. We shall establish the reciprocity (1.9) and $\left(1.9^{\prime}\right)$ by using convergence in mean square methods and also by ordinary convergence methods. The results are given in Theorems 1 and 2 below.

2. Two reference theorems. To prove Theorem 1 we would require an extended form of a Watson's Theorem [1, p. 221, Theorem 129 extended in the sense of $\$ 8.9$ on p. 226]. This theorem in the form in which it is going to be used here has been reproduced in an earlier paper of the author $[4, \S 2$, pp. 272-273]. In the present paper it will be referred to as Theorem A.

To prove Theorem 2 we shall use another result proved by Hardy and Titchmarsh $[7$, p. 141]. This result has also been reproduced in a desired 
form in an earlier paper of the author $[3, \S 2$, p. 20$]$. In the present paper it will be referred to as Theorem $B$.

3. Convergence in mean square. Here we shall use Theorem $\mathrm{A}$ to establish the reciprocity (1.9) and $\left(1.9^{\prime}\right)$.

First of all we estimate the asymptotic behaviour of $\mathfrak{S}^{(1)}(s)$ and $\mathfrak{S}^{(2)}(s)$, $s=\sigma+i t, \sigma$ and $t$ real, when $|t|$ is large. For large $s$ the asymptotic expansion $[8$, p. 278] of the Gamma function is given by

$$
\log \Gamma(s+a)=\left(s+a-\frac{1}{2}\right) \log s-s+\frac{1}{2} \log (2 \pi)+O\left(s^{-1}\right),
$$

where $|\arg s|<\pi$. To obtain the behaviour of $\mathfrak{S}^{(1)}(s)$ and $\mathfrak{S}^{(2)}(s)$ for large $|t|$, we replace Gamma functions involving $-s$ into those containing $+s$ with the help of the relation $[8$, p. 239]

$$
\Gamma(z) \Gamma(1-z)=\pi \operatorname{Cosec} \pi z .
$$

Then using (3.1) and the assumptions (1.5), (i) - (iv), we get and

$$
\mathfrak{Q}^{(1)}(s) x^{-s}=|t|^{\nu(\sigma-1 / 2)} \exp \{i t(D \log |t|-\log x-B)\}\left\{Q_{1}+O\left(|t|^{-1}\right)\right\},
$$

$$
\mathfrak{S}^{(2)}(s) x^{-s}=|t|^{\nu(\sigma-1 / 2)} \exp \{i t(D \log |t|-\log x-B)\}\left\{Q_{2}+O\left(|t|^{-1}\right)\right\},
$$

for large $|t|$, where $B$ is a constant and $Q_{1}$ and $Q_{2}$ are also constants each having one value for large positive $t$ and another for large negative $t$.

From (3.2) and (3.2') it follows that if $\sigma<\frac{1}{2}$ the integrals (1.6) and (1.6') are uniformly convergent for all $x$. We may therefore integrate with respect to $x$ under the integral sign. Let us denote

$$
\begin{aligned}
& H_{1}^{(1)}(x)=\int_{0}^{x} H^{(1)}(x) d x, \\
& H_{1}^{(2)}(x)=\int_{0}^{x} H^{(2)}(x) d x .
\end{aligned}
$$

Then

$$
\begin{aligned}
& H_{1}^{(1)}(x)=\frac{1}{2 \pi i} \int_{T} \frac{\mathfrak{S}^{(1)}(s)}{1-s} x^{1-s} d s, \\
& H_{1}^{(2)}(x)=\frac{1}{2 \pi i} \int_{T} \frac{\mathfrak{S}^{(2)}(s)}{1-s} x^{1-s} d s .
\end{aligned}
$$

These have been proved to be valid only when $\sigma<\frac{1}{2}$. But in the following theorem the contour $T$ will be taken along the line $\sigma=\frac{1}{2}$ and so it is necessary to have (3.4) and $\left(3.4^{\prime}\right)$ true for $\sigma=\frac{1}{2}$ also. 
We note that on a large circle whose points have polar coordinates $(\rho, \theta)$ the dominant factor in each of $\mathfrak{S}^{(1)}(s)$ and $\mathfrak{S}^{(2)}(s)$ is

$$
\exp \{D(\rho \log \rho) \cos \theta\} \text {. }
$$

Since $D>0$ we may close the contour $T$ in (1.6) by a large semicircle on the left. We can then express $H^{(1)}(x)$ as the sum of the residues of the integrand $\mathfrak{S}^{(1)}(s) x^{-s}$ at the poles of $\Gamma\left(c_{j}+\gamma_{j}\left(s-\frac{1}{2}\right)\right), j=1, \cdots, m$. For $H^{(1)}(x)$, we thus obtain $m$ power series, each of which is an entire function.

On multiplying (3.2) by $1 / t$, we see that the integral (3.4) converges when $\sigma=\frac{1}{2}$. Again (3.5) is the dominant factor for the integrand of (3.4) on a large circle so that the contour $T$ in (3.4) can be closed by a large semicircle on the left. The integral in (3.4) is the sum of the residues of

$$
\mathfrak{S}^{(1)}(s) x^{1-s} /(1-s)
$$

at the poles of $\Gamma\left(c_{j}+\gamma_{j}\left(s-\frac{1}{2}\right)\right), j=1, \cdots, m$. It is easily found that the residues are the terms of $H^{(1)}(x)$ each integrated from 0 to $x$. Since the power series in $H^{(1)}(x)$ are all entire functions such term by term integration is justified and so (3.3) and (3.4) are true even when $\sigma=\frac{1}{2}$. Similarly $\left(3.3^{\prime}\right)$ and $\left(3.4^{\prime}\right)$ are true when $\sigma=\frac{1}{2}$.

On the line $\sigma=\frac{1}{2}$, it is clear from (3.2) that $\mathfrak{S}^{(1)}(s) x^{-s}$ is bounded for all values of $t$. Hence on $\sigma=\frac{1}{2}, \quad \mathfrak{S}^{(1)}(s) x^{-s} /(1-s)$ belongs to $L^{2}\left(\frac{1}{2}-i \infty, \frac{1}{2}+i \infty\right)$ and so the integral in (3.4) converges in the mean square. Consequently, we may evaluate the integral in (3.4) either by the ordinary convergence methods or by the mean square convergence methods and the results must be the same, except for a set of points of measure zero. It has been seen above that the integral in (3.4) converges in the ordinary sense to $H_{1}^{(1)}(x)$ of (3.3). Hence if the integral in (3.4) is evaluated by the methods of mean square convergence the result will be $H_{1}^{(1)}(x)$ of (3.3). Similarly,

$$
\mathfrak{S}^{(2)}(s) x^{-s} /(1-s)
$$

belongs to $L^{2}\left(\frac{1}{2}-i \infty, \frac{1}{2}+i \infty\right)$ and the integral in (3.4') converges in mean square to $H_{1}^{(2)}(x)$ of $\left(3.3^{\prime}\right)$.

Theorem 1. If

$$
\begin{gathered}
m-q=n-p>0, \\
\alpha_{j}>0, \quad j=1, \cdots, p ; \quad \beta_{j}>0, \quad j=1, \cdots, q ; \\
\gamma_{j}>0, \quad j=1, \cdots, m ; \quad \delta_{j}>0, \quad j=1, \cdots, n, \\
\frac{1}{2} D=\sum^{m} \gamma_{j}-\sum^{q} \beta_{j}=\sum^{n} \delta_{j}-\sum^{p} \alpha_{j}>0, \\
\sum^{m} c_{j}-\sum^{q} b_{j}=\sum^{n} d_{j}-\sum^{p} a_{j},
\end{gathered}
$$




$$
\mathrm{Rl}\left(a_{j}\right)>0, \quad j=1, \cdots, p ; \quad \operatorname{Rl}\left(b_{j}\right)>0, \quad j=1, \cdots, q ;
$$

$$
\mathrm{Rl}\left(c_{j}\right)>0, \quad j=1, \cdots, m ; \quad \operatorname{Rl}\left(d_{j}\right)>0, \quad j=1, \cdots, n,
$$

$$
f(x) \text { belongs to } L^{2}(0, \infty) \text {, }
$$

then the formulae

$$
\begin{aligned}
& \frac{d}{d x} \int_{0}^{\infty} H_{1}^{(1)}(x u) f(u) \frac{d u}{u}=g^{(1)}(x), \\
& \frac{d}{d x} \int_{0}^{\infty} H_{1}^{(2)}(x u) f(u) \frac{d u}{u}=g^{(2)}(x),
\end{aligned}
$$

define almost everywhere functions $g^{(1)}(x)$ and $g^{(2)}(x)$ respectively both belonging to $L^{2}(0, \infty)$. Also the reciprocal formulae

$$
\begin{aligned}
& \frac{d}{d x} \int_{0}^{\infty} H_{1}^{(2)}(x u) g^{(1)}(u) \frac{d u}{u}=f(x), \\
& \frac{d}{d x} \int_{0}^{\infty} H_{1}^{(1)}(x u) g^{(2)}(u) \frac{d u}{u}=f(x),
\end{aligned}
$$

hold almost everywhere. And further

$$
\int_{0}^{\infty}[f(x)]^{2} d x=\int_{0}^{\infty} g^{(1)}(x) g^{(2)}(x) d x .
$$

In (3.4) and (3.4') which define $H_{1}^{(1)}(x)$ and $H_{1}^{(2)}(x)$ in a sense similar to that in (2.3) and (2.3') of Theorem A, we take $\sigma=\frac{1}{2}$ so that the contour $T$ is the line from $\frac{1}{2}-i \infty$ to $\frac{1}{2}+i \infty$. The conditions (v) ensure that the poles of $\mathfrak{S}^{(1)}(s)$ and $\mathfrak{S}^{(2)}(s)$ lie on such sides of $T$ as is required for the definition of $H^{(1)}(x)$ and $H^{(2)}(x)$.

We will now establish the truth of the requirements of Theorem $A$. The first requirement is that $\mathfrak{S}^{(1)}\left(\frac{1}{2}+i t\right)$ and $\mathfrak{S}^{(2)}\left(\frac{1}{2}+i t\right)$ are bounded functions of $t$ satisfying (1.8) with $s=\frac{1}{2}+i t$. This is clearly true from (3.2), (3.2') and (1.7), (1.7 ). The second requirement is that $H_{1}^{(1)}(x)$ and $H_{1}^{(2)}(x)$ are related with $\mathfrak{S}^{(1)}(s)$ and $\mathfrak{S}^{(2)}(s)$, the Mellin transforms of $H^{(1)}(x)$ and $H^{(2)}(x)$ respectively, according to (2.3) and $\left(2.3^{\prime}\right)$ of Theorem A. This is explained just before the enunciation of the present theorem. The third requirement that $f(x)$ belongs to $L^{2}(0, \infty)$ is covered by the hypothesis (vi) of the theorem.

Since all the conditions of Theorem $A$ are satisfied, its conclusions follow and in our case the conclusions are (3.6), (3.6'), (3.7), (3.7') and (3.8).

4. Convergence in the ordinary sense. As is well known in the theory of Fourier transform, theorems using the ordinary convergence methods appear to be much more difficult than those which use convergence in mean square methods. 
THEOREM 2. If

(i)

$$
m-q=n-p>0,
$$

(ii)

$$
\alpha_{j}>0, \quad j=1, \cdots, p ; \quad \beta_{j}>0, \quad j=1, \cdots, q ;
$$$$
\gamma_{j}>0, \quad j=1, \cdots, m ; \quad \delta_{j}>0, \quad j=1, \cdots, n,
$$

$$
\frac{1}{2} D=\sum^{m} \gamma_{j}-\sum^{q} \beta_{j}=\sum^{n} \delta_{j}-\sum^{p} \alpha_{j}>0,
$$

$$
\sum^{m} c_{j}-\sum^{q} b_{j}=\sum^{n} d_{j}-\sum^{p} a_{j},
$$

(v) $\quad \operatorname{Rl}\left(a_{j}\right)>\alpha_{j} / 2 D, \quad j=1, \cdots, p ; \quad \operatorname{Rl}\left(b_{j}\right)>\beta_{j} / 2 D, j=1, \cdots, q$;

(vi) $\quad \mathrm{Rl}\left(c_{j}\right) \geqq \gamma_{j} / 2 D, \quad j=1, \cdots, m ; \quad \operatorname{Rl}\left(d_{j}\right) \geqq \delta_{j} / 2 D, j=1, \cdots, n$,

(vii) $y^{(1-D) / 2 D} f(y)$ belongs to $L(0, \infty)$ and $f(y)$ is of bounded variation near $y=x(x>0)$,

then

$$
\int_{0}^{\infty} H^{(1)}(x u)\left\{\int_{0}^{\infty} H^{(2)}(u y) f(y) d y\right\} d u=\frac{1}{2}\{f(x+0)+f(x-0)\} .
$$

We first transform (4.1) into a form to which Theorem B can be applied. For the sake of brevity, let us define a quantity $\mu$ by

$$
\mu=\frac{\prod^{m}\left(\gamma_{j} / D\right)^{\gamma_{j} / D} \prod^{n}\left(\delta_{j} / D\right)^{\delta_{j} / D}}{\prod^{p}\left(\alpha_{j} / D\right)^{\alpha_{j} / D} \prod^{q}\left(\beta_{j} / D\right)^{\beta_{j} / D}} \equiv \frac{1}{D}\left\{\frac{\prod^{m} \gamma_{j}^{\gamma_{j}} \prod^{n} \delta_{j}^{\delta_{j}}}{\prod^{p} \alpha_{j}^{\alpha_{j}} \prod^{q} \beta_{j}^{\beta_{j}}}\right\}^{(1 / \nu)} .
$$

Since all the terms on the right-hand side of (4.2) are positive, $\mu$ is also positive. We now write

$$
x=X^{\nu}, \quad y=Y^{\nu}, \quad u=\left(U_{\mu}\right)^{\nu}
$$

in (4.1). By an easy computation and adjustments, it takes the form

$$
\int_{0}^{\infty} K^{(1)}(X U)\left\{\int_{0}^{\infty} K^{(2)}(Y U) f^{*}(Y) d Y\right\} d U=\frac{1}{2}\left\{f^{*}(X+0)+f^{*}(X-0)\right\},
$$

where

$$
\begin{aligned}
K^{(1)}(X) & =H^{(1)}\left\{\left(X_{\mu}\right)^{\nu}\right\}\left(X_{\mu}\right)^{(\nu-1) / 2} \mu^{1 / 2} D, \\
K^{(2)}(X) & =H^{(2)}\left\{\left(X_{\mu}\right)^{\nu}\right\}\left(X_{\mu}\right)^{(\nu-1) / 2} \mu^{1 / 2} D, \\
f^{*}(Y) & =Y^{(\nu-1) / 2} f\left(Y^{\nu}\right) .
\end{aligned}
$$


Our object is to prove (4.1) by proving (4.4) first in which the kernels are $K^{(1)}(X)$ and $K^{(2)}(X)$. The reasons for making the above transformations will appear in $\$ 7$ below. From (1.4), we have

$$
\text { (4.7) } K^{(1)}(X)=\frac{1}{2 \pi i} \int_{T} \frac{\prod^{m} \Gamma\left(c_{j}+\gamma_{j}\left(s-\frac{1}{2}\right)\right) \stackrel{p}{\prod} \Gamma\left(a_{j}-\alpha_{j}\left(s-\frac{1}{2}\right)\right)}{\prod^{n} \Gamma\left(d_{j}-\delta_{j}\left(s-\frac{1}{2}\right)\right) \stackrel{q}{\prod} \Gamma\left(b_{j}+\beta_{j}\left(s-\frac{1}{2}\right)\right)} \cdot \frac{\left(X_{\mu}\right)^{-D s+(D-1) / 2} \mu^{1 / 2} D d s,}{}
$$

where the contour $T^{\prime}$ of integration is a straight line parallel to the imaginary axis in the $s$-plane separating the poles of $\Gamma\left(c_{j}+\gamma_{j}\left(s-\frac{1}{2}\right)\right), j=1, \cdots, m$ and $\Gamma\left(a_{j}-\alpha_{j}\left(s-\frac{1}{2}\right)\right), j=1, \ldots, p$.

Making one more transformation

$$
-D s+(D-1) / 2=-S,
$$

we get

$$
K^{(1)}(X)=\frac{1}{2 \pi i} \int_{T} \Re^{(1)}(S) X^{-S} d S,
$$

where

$$
=\frac{\prod^{m} \Gamma\left(c_{j}+\gamma_{j}\left(s-\frac{1}{2}\right) / D\right) \stackrel{\prod^{(1)}}{\prod} \Gamma\left(a_{j}-\alpha_{j}\left(S-\frac{1}{2}\right) / D\right)}{\prod^{n} \Gamma\left(d_{j}-\delta_{j}\left(S-\frac{1}{2}\right) / D\right) \prod_{\prod}^{q} \Gamma\left(b_{j}+\beta_{j}\left(S-\frac{1}{2}\right) / D\right)} \mu^{1 / 2-s},
$$

and the contour $T$ is any straight line parallel to the imaginary axis in the $S(=\sigma+i t)$ plane for which $0<\sigma<\frac{1}{2}$. The corresponding contour $T^{\prime}$ for (4.7) is easily deduced from this statement by means of the transformation (4.8). Similarly, we have

$$
K^{(2)}(X)=\frac{1}{2 \pi i} \int_{T} \boldsymbol{I}^{(2)}(S) X^{-S} d S,
$$

where

$$
=\frac{\prod^{\Re^{(2)}} \Gamma\left(d_{j}+\delta_{j}\left(S-\frac{1}{2}\right) / D\right) \prod^{q} \Gamma\left(b_{j}-\beta_{j}\left(S-\frac{1}{2}\right) / D\right)}{\prod^{m} \Gamma\left(c_{j}-\gamma_{j}\left(S-\frac{1}{2}\right) / D\right) \prod^{p} \Gamma\left(a_{j}+\alpha_{j}\left(S-\frac{1}{2}\right) / D\right)} \mu^{1 / 2-S} .
$$


Evidently, $\Omega^{(1)}(S)$ and $\Omega^{(2)}(S)$ are the Mellin transforms of $K^{(1)}(X)$ and $K^{(2)}(X)$ respectively.

We shall now use Theorem B to establish (4.4). It will be shown that $\Omega^{(1)}(S)$ and $\Omega^{(2)}(S)$ satisfy conditions (i), (ii) and (iii) and $f^{*}(Y)$ satisfies condition (iv) of that theorem.

Let $S=\sigma+i$. The poles of $\mathscr{\imath}^{(1)}(S)$ are at the points

$$
\begin{array}{ll}
\frac{1}{2}-D\left(r+c_{j}\right) / \gamma_{j}, & j=1, \cdots, m, \\
\frac{1}{2}+D\left(r+a_{j}\right) / \alpha_{j}, & j=1, \cdots, p,
\end{array}
$$

and those of $\Re^{(2)}(S)$ are at the points

$$
\begin{array}{ll}
\frac{1}{2}-D\left(r+d_{j}\right) / \delta_{j}, & j=1, \cdots, n, \\
\frac{1}{2}+D\left(r+b_{j}\right) / \beta_{j}, & j=1, \cdots, q,
\end{array}
$$

where $r$ is a positive integer or zero. From conditions (v) of the Theorem 2, it follows that all the $a_{\text {j }}$ poles of $\Omega^{(1)}(S)$ and $b_{j}$-poles of $\Omega^{(2)}(S)$ lie on the right of $\sigma=1$. In conditions (vi) if only the inequalities hold then all the $c_{j}$-poles of $\Omega^{(1)}(S)$ and $d_{j}$-poles of $\Omega^{(2)}(S)$ lie on the left of $\sigma=0$. If, however, the equality holds for some of the $c_{j}$ 's and $d_{j}$ 's in (vi), then there are poles of $\Omega^{(1)}(S)$ and $\Omega^{(2)}(S)$ on the imaginary axis and there can be at the most $m$ of $\Omega^{(1)}(S)$ and $n$ of $\Omega^{(2)}(S)$. Since the only singularities of $\Omega^{(1)}(S)$ and $\Omega^{(2)}(S)$ are isolated simple poles it follows that $\sigma_{0}<0$ and $\sigma_{1}>1$ can be found such that $\Omega^{(1)}(S)$ and $\Omega^{(2)}(S)$ are both regular in the strip $\sigma_{0}<\sigma<\sigma_{1}$ except possibly for a finite number of simple poles on the imaginary axis.

To prove that $\Omega^{(1)}(S)$ and $\Omega^{(2)}(S)$ also satisfy the second requirement for being of class $k^{\prime}$ we shall examine the asymptotic behaviour of $\AA^{(1)}(S)$ and $\Omega^{(2)}(S)$ for large positive and negative $t$.

In the expression for $\Re^{(1)}(S)$, let the Gamma functions involving $-S$ be changed into the Gamma functions containing $+S$ by using the formula $\left(3.1^{\prime}\right)$ so that we get

$$
\begin{aligned}
\boldsymbol{\Omega}^{(1)}(S)= & \frac{\prod^{m} \Gamma\left(c_{j}+\gamma_{j}\left(S-\frac{1}{2}\right) / D\right) \prod^{n} \Gamma\left(1-d_{j}+\delta_{j}\left(S-\frac{1}{2}\right) / D\right)}{\prod^{p} \Gamma\left(1-a_{j}+\alpha_{j}\left(S-\frac{1}{2}\right) / D\right) \prod^{q} \Gamma\left(b_{j}+\beta_{j}\left(S-\frac{1}{2}\right) / D\right)} \\
& \cdot \frac{\prod^{n} \sin \pi\left(d_{j}-\delta_{j}\left(S-\frac{1}{2}\right) / D\right)}{\prod^{p} \sin \pi\left(a_{j}-\alpha_{j}\left(S-\frac{1}{2}\right) / D\right)} \pi^{p-n} \mu^{1 / 2-S} .
\end{aligned}
$$


To the factors in (4.13) involving Gamma functions only, we now apply (3.1). On using the conditions (i) through (iv) of the Theorem 2 and (4.2), we get for large $|S|$ and $|\arg S| \leqq \pi-\delta, \delta>0$, the contribution to the asymptotic expansion of $\boldsymbol{\Omega}^{(1)}(S)$ due to the factors involving Gamma functions only as

$$
\mu^{S-1 / 2} \exp \left\{\left(S-\frac{1}{2}\right) \log S-S\right\}\left\{A_{1}+B_{1} / S+O\left(|S|^{-2}\right)\right\},
$$

where $A_{1}$ and $B_{1}$ are constants independent of $S$. Using (3.1) again this expression is equivalent to

$$
\mu^{S-1 / 2} \Gamma(S)\left\{A_{2}+B_{2} / S+O\left(|S|^{-2}\right)\right\},
$$

where $A_{2}$ and $B_{2}$ are constants.

Next we consider the contribution to the asymptotic expansion of $\Omega^{(1)}(S)$ due to the factors involving sin functions. Let $S=\sigma+i$. Allowing $|t|$ become large it is easily seen that

$$
\frac{\sin \pi\left(d_{j}-\delta_{j}\left(S-\frac{1}{2}\right) / D\right)}{\left(\cos \frac{1}{2} S \pi\right)^{2 \delta_{j} / D}}=E_{1}+O\left(e^{-|t|}\right),
$$

where $E_{1}$ is a constant such that

$$
E_{1}=2^{22_{j} / D-1} \exp \left\{i \pi\left(d_{j}-\frac{1}{2}+\delta_{j} / 2 D\right)\right\} \text { for large positive } t
$$

and

$$
E_{1}=2^{2 \delta_{j} / D-1} \exp \left\{-i \pi\left(d_{j}-\frac{1}{2}+\delta_{j} / 2 D\right)\right\} \quad \text { for large negative } t .
$$

There are $n \sin$ factors in the numerator and $p$ in the denominator of (4.13) and the total contribution of all the sin factors in (4.13) to the asymptotic expansion of $\boldsymbol{\Omega}^{(1)}(S)$ is of the form

$$
\left\{E_{2}+O\left(e^{-|t|}\right)\right\} \cos \frac{1}{2} S \pi,
$$

where $E_{2}$ is a constant having one value for large positive $t$ and the conjugate value for large negative $t$.

The asymptotic expansion of $\Omega^{(1)}(S)$ can now be written by taking the product of (4.14), (4.15) and the factors $\pi^{n-p} \mu^{1 / 2-S}$ We thus have finally the following result. For $S=\sigma+i t$ and sufficiently large values of $|t|$ the asymptotic expansion of $\Omega^{(1)}(S)$ is given by 


$$
\mathscr{R}^{(1)}(S)=\Gamma(S) \cos \left(\frac{1}{2} S \pi\right)\left\{\mathscr{A}_{1}+\mathfrak{B}_{1} / S+O\left(|s|^{-2}\right)\right\},
$$

where the constants $\mathfrak{A}_{1}$ and $\mathfrak{B}_{1}$ each have one value for large positive $t$ and another value for large negative $t$.

In an exactly similar manner it is seen that for large values of $|t|$,

$$
\mathfrak{R}^{(2)}(S)=\Gamma(S) \cos \left(\frac{1}{2} S \pi\right)\left\{\mathscr{A}_{2}+\mathfrak{B}_{2} / S+O\left(|s|^{-2}\right)\right\},
$$

where $\mathfrak{A}_{2}$ and $\mathfrak{B}_{2}$ are constants each having one value for large positive $t$ and another for large negative $t$.

This establishes that $\Omega^{(1)}(S)$ and $\Omega^{(2)}(S)$ both belong to the class $k^{\prime}$.

The third condition of Theorem $B$ is obviously satisfied by $\Omega^{(1)}(S)$ and $\Omega^{(2)}(S)$ if we look at their forms in (4.10) and (4.12).

The fourth condition is that $f^{*}(Y)$ belongs to $L(0, \infty)$ and that it is of bounded variation near $Y=X(X>0)$. This requires that

$$
\int_{0}^{\infty}\left|Y^{(\nu-1) / 2} f\left(Y^{\nu}\right)\right| d Y
$$

must exist and the integrand must be of bounded variation near $Y=X$ $(X>0)$. On putting $Y^{\nu}=y$, it follows that these two requirements are fulfilled by the enunciation (vii) of the theorem.

All the requirements of Theorem $B$ have thus been shown to be fulfilled. The conclusion of that theorem must therefore follow and consequently the equation (4.4) is true. On using the transformation (4.3) in the reverse sense it follows that (4.1) is true.

5. Discontinuous integrals associated with $H^{(1)}(x)$ and $H^{(2)}(x)$.

THEOREM 3. Let $H^{(1)}(x), H^{(2)}(x)$ be defined by (1.4), $\left(1.4^{\prime}\right)$ and $H_{1}^{(1)}(x)$, $H_{1}^{(2)}(x)$ by (3.3), (3.3'). If the conditions (i) through (vi) of Theorem 2 hold, then

$$
\int_{0}^{\infty} H^{(1)}(x u) H_{1}^{(2)}(u y) \frac{d u}{u}= \begin{cases}0, & x>y>0, \\ \frac{1}{2}, & x=y>0, \\ 1, & y>x>0 .\end{cases}
$$

In Theorem 2, take $f(y)$ defined by

$$
f(y)= \begin{cases}1, & y<Y, \\ 0, & y>Y,\end{cases}
$$

so that 


$$
\frac{1}{2}\{f(x+0)+f(x-0)\}= \begin{cases}1, & 0<x<Y \\ \frac{1}{2}, & x=Y>0 \\ 0, & x>Y>0\end{cases}
$$

We have

$$
\int_{0}^{\infty} H^{(2)}(u y) f(y) d y=\int_{0}^{Y} H^{(2)}(u y) d y=\frac{H_{1}^{(2)}(u Y)}{u} .
$$

(5.1), then, follows from Theorem 2.

6. The asymptotic expansion of $H^{(1)}(x)$ and $H^{(2)}(x)$ for positive real $x$. In this section we give a theorem concerning the asymptotic expansion of $H^{(1)}(x)$ and $H^{(2)}(x)$. We are confined to the case when $x$ is real and positive as the investigation for complex $x$ becomes too involved.

Corresponding to a given $\tilde{\omega}$, an asymptotic expansion for $H^{(1)}(x)$ and $H^{(2)}(x)$, when $x$ is large and positive, with an error term $O\left(x^{-\tilde{\omega}}\right)$, has been found.

Theorem 4. Assumptions:

$$
\begin{gathered}
m-q=n-p>0, \\
\alpha_{j}>0, \quad j=1, \cdots, p, \quad \beta_{j}>0, \quad j=1, \cdots, q ; \\
\gamma_{j}>0, \quad j=1, \cdots, m, \quad \delta_{j}>0, \quad j=1, \cdots, n, \\
\frac{1}{2} D=\sum^{m} \gamma_{j}-\sum^{q} \beta_{j}=\sum^{n} \delta_{j}-\sum^{p} \alpha_{j}>0, \\
\frac{1}{2} L=\sum^{m} c_{j}-\sum^{q} b_{j}=\sum^{n} d_{j}-\sum^{p} a_{j},
\end{gathered}
$$
$x$ is real and positive,

(vii) $\quad \operatorname{Rl}\left(c_{j}\right)>\gamma_{j} / 2, \quad j=1, \cdots, m, \quad \operatorname{Rl}\left(d_{j}\right)>\delta_{j} / 2, \quad j=1, \cdots, n$,

(viii) given $\widetilde{\omega}>\frac{1}{2}, \quad N$ denotes the greatest integer less than $\left\{D\left(\widetilde{\omega}-\frac{1}{2}\right)+3 / 2\right\}, M_{j}$ denotes the greatest positive integer less than $\left\{\alpha_{j}\left(\widetilde{\omega}-\frac{1}{2}\right)-\operatorname{Rl}\left(a_{j}\right)\right\}, j=1, \ldots, p$ and $M_{j}^{\prime}$ denotes the greatest positive integer less than $\left\{\beta_{j}\left(\widetilde{\omega}-\frac{1}{2}\right)-\operatorname{Rl}\left(b_{j}\right)\right\}, j=1, \ldots, q$,

(ix)

$$
m-q=n-p \text { is an odd positive integer. }
$$

Conclusions: 


$$
H^{(1)}(x)=\frac{1}{D}\left(\frac{x}{\beta}\right)^{(1-D) / 2 D} \sum_{j=0}^{N} \nu_{j}\left(\frac{x}{\beta}\right)^{-j / D} \sin \left\{\left(L+\frac{1}{2}-j\right) \pi / 2-\left(\frac{x}{\beta}\right)^{1 / D}\right\}
$$

$$
\begin{aligned}
& +\sum_{j=1}^{p} x^{-1 / 2-a_{j} / \alpha_{j}}\left(A_{j}+B_{j} x^{-1 / \alpha_{j}}+C_{j} x^{-2 / \alpha_{j}}+\cdots+U_{j} x^{-M_{j} / \alpha_{j}}\right) \\
& +O\left(x^{-\tilde{\omega}}\right)
\end{aligned}
$$

and

$$
H^{(2)}(x)=\frac{1}{D}\left(\frac{x}{\beta}\right)^{(1-D) / 2 D} \sum_{j=0}^{N} \nu_{j}^{\prime}\left(\frac{x}{\beta}\right)^{-j / D} \sin \left\{\left(L+\frac{1}{2}-j\right) \pi / 2-\left(\frac{x}{\beta}\right)^{1 / D}\right\}
$$

$$
\begin{aligned}
& +\sum_{j=1}^{q} x^{-1 / 2-b_{j} / \beta_{j}}\left(A_{j}^{\prime}+B_{j}^{\prime} x^{-1 / \beta_{j}}+C_{j}^{\prime} x^{-2 / \beta_{j}}+\cdots+U_{j}^{\prime} x^{-M_{j}^{\prime} / \beta_{j}}\right) \\
& +O\left(x^{-\tilde{\omega}}\right),
\end{aligned}
$$

where

$$
\beta=\mu^{D},
$$

and

$$
\begin{gathered}
\boldsymbol{\nu}_{j}, \boldsymbol{\nu}_{j}^{\prime}, \quad j=0,1, \cdots, N, \\
A_{j}, B_{j}, C_{j}, \cdots, U_{j}, \quad j=1, \cdots, p, \\
A_{j}^{\prime}, B_{j}^{\prime}, C_{j}^{\prime}, \cdots, U_{j}^{\prime}, \quad j=1, \cdots, q,
\end{gathered}
$$

are constants which depend on the parameters

$$
\begin{array}{llll}
a_{j}, \alpha_{j}, & j=1, \cdots, p, & b_{j}, \beta_{j}, & j=1, \cdots, q, \\
c_{j}, \gamma_{j}, & j=1, \cdots, m, & d_{j}, \delta_{j}, & j=1, \cdots, n,
\end{array}
$$

but are independent of $x$.

If $m-q=n-p$ is an even positive integer (instead of the condition (ix) above) then in the right hand side of (6.1) and (6.2) we must replace $\sin$ by cos.

This theorem has been obtained following the lines of a similar theorem of Fox [5, p.417] concerning a symmetrical Fourier kernel. The proof is, therefore, being omitted. The constants (6.4) are computed by a method specified in Lemma 6 of Fox's paper [5, p.423] and the constants $\left(6.4^{\prime}\right)$ are computed by calculating residues as specified in $\$ 12$ of the same paper [ 5 , p. 427].

7. The asymptotic expansions of $H^{(1)}(x)$ and $H^{(2)}(x)$ enable us to explain the need for the transformations (4.3) which bring forth the new kernels 
$K^{(1)}(x)$ and $K^{(2)}(x)$ of (4.5) and $\left(4.5^{\prime}\right)$.

The classical Fourier kernels such as $\sin x, \cos x$ and $\sqrt{ }(x) J_{\nu}(x)$ all behave in a very similar manner as $x \rightarrow \infty$. When $x$ is complex they all tend to infinity with exponential rapidity and when $x$ is real they all oscillate finitely as $x \rightarrow \infty$ just like $\cos x$. It is in fact the behaviour of a function on the real axis which decides whether or not it is a Fourier kernel.

If we look at equations (6.1) and (6.2), then, by the conditions (v) of Theorem 2, the algebraic terms in the asymptotic expansions of $H^{(1)}(x)$ and $H^{(2)}(x)$ tend to zero as $x \rightarrow \infty$. But the trigonometric terms contain a factor $x^{(1-D) / 2 D}$. Hence, when $D \neq 1, H^{(1)}(x)$ and $H^{(2)}(x)$ cannot oscillate finitely, as $x \rightarrow \infty$. On the other hand, (6.1) and (6.2) show that $K^{(1)}(X)$ and $K^{(2)}(X)$, (4.5) and (4.5'), contain algebraic terms which tend to zero as $x$ approaches infinity, but that the trigonometric terms are of the type

$$
\mu^{D / 2} \sum_{j=0}^{N} \nu_{j} X^{-j} \sin \left\{\left(L+\frac{1}{2}-j\right) \pi / 2-X\right\} .
$$

Evidently, the terms in (7.1) for which $j \geqq 1$ all tend to zero as $X$ tends to infinity but the term corresponding to $j=0$ oscillates finitely as $X \rightarrow \infty$. Hence the kernels $K^{(1)}(X)$ and $K^{(2)}(X)$ behave much like the Fourier kernels of classical theory and so much of this theory can be applied to them. This explains the need for the transformations (4.3).

\section{REFERENCES}

1. E. C. Titchmarsh, Introduction to the theory of Fourier integrals, Oxford Univ. Press, Oxford, 1937.

2. Roop Narain, The G-functions as unsymmetrical Fourier kernels. I, Proc. Amer. Math. Soc. 13 (1962), 950-959.

3. - The G-functions as unsymmetrical Fourier kernels. II, Proc. Amer. Math. Soc. 14 (1963), 18-28.

4. _ The G-functions as unsymmetrical Fourier kernels. III, Proc. Amer. Math. Soc. 14 (1963), 271-277.

5. C. Fox, The $G$ and $H$ functions as symmetrical Fourier kernels, Trans. Amer. Math. Soc. 98 (1961), 395-429.

6. J. Boersma, On a function, which is a special case of Meijer's G-function, Compositio Math. 15 (1962), 34-63.

7. G. H. Hardy and E. C. Titchmarsh, A class of Fourier kernels, Proc. London Math. Soc. Ser. 235 (1933), 116-155.

8. E. T. Whittaker and G. N. Watson, A course of modern analysis, Cambridge Univ. Press, Cambridge, 1935.

Wayne State University, DETROIT, MiCHIGAN 